\title{
Robust Regulation and Tracking Control of a Class of Uncertain 2DOF Underactuated Mechanical Systems
}

\author{
David I. Rosas Almeida, Carlos Gamez, and Raul Rascón \\ Engineering Faculty, Autonomous University of Baja California, 21900 Mexicali, BC, Mexico \\ Correspondence should be addressed to David I. Rosas Almeida; drosas@uabc.edu.mx
}

Received 15 May 2015; Accepted 2 August 2015

Academic Editor: Wenguang Yu

Copyright ( 2015 David I. Rosas Almeida et al. This is an open access article distributed under the Creative Commons Attribution License, which permits unrestricted use, distribution, and reproduction in any medium, provided the original work is properly cited.

\begin{abstract}
A strategy to design and implement a robust controller for a class of underactuated mechanical systems, with two degrees of freedom, which solves the problems of regulation and trajectory tracking, is proposed. This control strategy considers the partial measurement of the state vector and the presence of parametric uncertainties in the plant; these conditions are common in the implementation of a control system. The strategy is based on the use of robust finite time convergence observers to estimate the unmeasured state variables, unknown disturbances, and other signals needed for the control system implementation. The performance of the control strategy is illustrated numerically and experimentally.
\end{abstract}

\section{Introduction}

Antecedents and Motivation. Control of underactuated mechanical systems, systems with fewer number of control inputs than their degrees of freedom, has received much attention in the last decades. This is because of the theoretical challenges as well as practical applicability; robots, aerospace vehicles, underwater vehicles, and surface vessels are some examples of underactuated mechanical systems. Some important papers which address this control problem for different situations are [1-9]. While many interesting techniques and results have been presented for this class of systems, the control of them still remains an open problem. Important issues are as follows: how control models can be formulated for such systems and how closed-loop control problems can be solved and implemented. These issues are addressed in this paper for a particular class of uncertain underactuated mechanical systems. These problems have been addressed by many authors and important solutions have been proposed, some of which are as follows.

In [10] a sliding mode control method for a class of second-order underactuated mechanical systems is proposed; the controller has the double-layer structure. Firstly, the system states are divided into several different subsystems. For each of these subsystems, a first-layer sliding plane is constructed; from that, a second-layer sliding plane is constructed. By analyzing the features of the model of the plant, they derive the sliding control law. Here, the proposed controller only solves the regulation problem; furthermore, the implementation of the controller requires the measurement of full state vector; this condition is not satisfied in practice. For a similar class of systems, in [11], the Olfati transformation is applied first to represent the system into a special cascade form. Since, in general cases, some of the terms in the new space might become too complex to drive, they are regarded as uncertainties. A backstepping-like adaptive control based on function approximation technique is designed so that the system in the new space can be stabilized with uniformly ultimately bounded performance. This paper assumes knowledge of all system parameters and the measurement of all state variables and the perturbation terms that appear in the approach are well known, but experimental results are not presented.

Other works deal with particular systems, for example, $[1,3,12,13]$, but many of them only deal with the regulation problem and present performance results through numerical simulations. Reference [14] addresses the observer-based multivariable control of a class of nonlinear, underactuated Lagrangian systems with application to trajectory tracking and sway control of a 3D overhead gantry crane subject to 
Coulomb friction. A second-order sliding mode observer is used for the estimation of velocities. Based on these estimates, the sliding function of a second-order sliding mode controller for trajectory tracking and antiswing control is proposed. This is a very important paper because it considers a very common situation in practice, the lack of measurement of the velocities, but it only show numerical results.

An important work is presented in [15] where, for a class of second-order underactuated mechanical systems, a robust finite time control strategy is proposed. The robust finite time controller drives the tracking error to be zero at the fixed final time. By utilizing a Lyapunov stability theorem, the controller can achieve finite time tracking of desired reference signals for underactuated systems, which are subject to both external disturbances and system uncertainties. However, the complete measurement of the state vector is assumed and only stabilization problem is solved. Moreover, illustration controller performance is through numerical simulations.

Main Contribution. We propose a strategy to design and implement a robust controller for a class of underactuated mechanical systems, with two degrees of freedom, which solves the problems of regulation and trajectory tracking. This control strategy considers the partial measurement of the state vector and the presence of parametric uncertainties in the plant; these conditions are common in the implementation of a control system.

The strategy is based on the use of robust finite time convergence observers to estimate the unmeasured state variables, unknown disturbances, and other signals needed for the control system implementation. The performance of the control strategy is illustrated numerically and experimentally.

Paper Structure. This paper is organized as follows: Section 2 provides the control problem, the model of the plant, and the control objective. In Section 3, we propose the solution to the problem; to implement such solution is necessary to know the velocities, the exact value of the disturbances, and the availability of auxiliary signals and their derivatives, which are unknown. One way to implement this control signal is presented in Section 4, where with the help of robust observers with finite time convergence we estimate all the terms needed for implementation. Section 5 shows the performance of the controller through numerical simulations and experimental results. Finally, in Section 6, we present some general conclusions.

\section{Problem Statement}

Consider a 2DOF underactuated mechanical system whose dynamics are given by

$$
\begin{aligned}
& \ddot{q}_{1}=f_{1}\left(q_{1}, \dot{q}_{1}, q_{2}, \dot{q}_{2}\right)+g_{1}\left(q_{1}, q_{2}\right) u+\gamma_{1}(\cdot), \\
& \ddot{q}_{2}=f_{2}\left(q_{2}, \dot{q}_{2}\right)+g_{2} v\left(q_{1}\right)+\gamma_{2}(\cdot), \\
& y_{1}=q_{1}, \\
& y_{2}=q_{2},
\end{aligned}
$$

where $q_{1}, q_{2}, \dot{q}_{1}$, and $\dot{q}_{2}$ are the generalized positions and velocities, respectively. $f_{1}(\cdot), f_{2}(\cdot)$, and $g_{1}(\cdot)$ are smooth functions; $g_{1}(\cdot) \neq 0$ for all $q_{1}$ and $q_{2} \cdot g_{2} \neq 0$ is a constant, $v\left(q_{1}\right)$ is an invertible function for all $q_{1}$ in the domain of operation of the system, and $u$ is the control input. $\gamma_{1}(\cdot)$ and $\gamma_{2}(\cdot)$ are smooth terms due to parameter variations; based on Lagrangian model (1), these terms may depend on $q_{1}, q_{2}, \dot{q}_{1}$, $\dot{q}_{2}, \ddot{q}_{1}$, and $\ddot{q}_{2}$, but if these variables are bounded, $\gamma_{1}(\cdot)$ and $\gamma_{2}(\cdot)$ also are bounded [16]. Additionally, we considered that there is no measurement of the velocities $\dot{q}_{1}$ and $\dot{q}_{2}$.

Some well known mechanisms that belong to this class of underactuated systems are the mass-spring-damper, magnetic suspension, and the ball and beam systems.

A state space representation of system (1) is

$$
\begin{aligned}
& \dot{x}_{1}=x_{2}, \\
& \dot{x}_{2}=f_{1}\left(x_{1}, x_{2}, x_{3}, x_{4}\right)+g_{1}\left(x_{1}, x_{2}\right) u+\gamma_{1}(\cdot), \\
& \dot{x}_{3}=x_{4}, \\
& \dot{x}_{4}=f_{2}\left(x_{3}, x_{4}\right)+g_{2} v\left(x_{1}\right)+\gamma_{2}(\cdot), \\
& y_{1}=x_{1}, \\
& y_{2}=x_{3} .
\end{aligned}
$$

The control problem, for system (2), is to design a control input $u$ such that the underactuated position $x_{3}$ tracks a reference signal $y_{r}(t)$ in asymptotic form; in other words

$$
\lim _{t \rightarrow \infty}\left|y_{2}-y_{r}(t)\right|=0
$$

where $y_{r}(t)$ is a $\mathscr{C}^{k}$ function, for a sufficiently large $k$.

To solve the control problem we define the error variables $e_{1}=x_{3}-y_{r}(t)$ and $e_{2}=x_{4}-\dot{y}_{r}(t)$, whose dynamics are given by

$$
\begin{aligned}
\dot{e}_{1}= & e_{2}, \\
\dot{e}_{2}= & f_{2}\left(e_{1}+y_{r}, e_{2}+\dot{y}_{r}\right)+g_{2} v\left(x_{1}\right)+\gamma_{2}(\cdot)-\ddot{y}_{r}(t), \\
\dot{x}_{1}= & x_{2}, \\
\dot{x}_{2}= & f_{1}\left(x_{1}, x_{2}, e_{1}+y_{r}, e_{2}+\dot{y}_{r}\right)+g_{1}\left(x_{1}, x_{2}\right) u \\
& +\gamma_{1}(\cdot), \\
y_{e}= & e_{1}, \\
y_{1}= & x_{1} .
\end{aligned}
$$

Now we can say that the control problem is to design a control input $u$ such that the origin of the error variables of subsystems (4) and (5) will be an asymptotic stable equilibrium point, while the variables $x_{1}$ and $x_{2}$ stay bounded.

\section{Control Strategy}

In this section we present a strategy to solve the control problem considering that every disturbance terms and velocities are known; the next section will show its implementation. 
System ((4)-(9)) is formed by two subsystems; the unactuated part is

$$
\begin{aligned}
& \dot{e}_{1}=e_{2}, \\
& \dot{e}_{2}=f_{2}\left(e_{1}+y_{r}, e_{2}+\dot{y}_{r}\right)+g_{2} v\left(x_{1}\right)+\gamma_{2}(\cdot)-\ddot{y}_{r}(t), \\
& y_{e}=e_{1}
\end{aligned}
$$

and the actuated part is

$$
\begin{aligned}
\dot{x}_{1}= & x_{2}, \\
\dot{x}_{2}= & f_{1}\left(x_{1}, x_{2}, e_{1}+y_{r}, e_{2}+\dot{y}_{r}\right)+g_{1}\left(x_{1}, x_{2}\right) u \\
& \quad+\gamma_{1}(\cdot), \\
y_{1}= & x_{1} .
\end{aligned}
$$

The function $v\left(x_{1}\right)$ in (10) can be seen as a control input for this subsystem; therefore we rename $v\left(x_{1}\right)$ as $u_{e}$ or $x_{1}=$ $v^{-1}\left(u_{e}\right)$ in $(10)$ :

$$
\begin{aligned}
& \dot{e}_{1}=e_{2}, \\
& \dot{e}_{2}=f_{2}\left(e_{1}+y_{r}, e_{2}+\dot{y}_{r}\right)+\gamma_{2}(\cdot)-\ddot{y}_{r}(t)+g_{2} u_{e}, \\
& y_{e}=e_{1} .
\end{aligned}
$$

An ideal control $u_{e}$ that stabilizes the origin of system (12) is

$$
\begin{aligned}
u_{e} & =\frac{1}{g_{2}}\left(-f_{2}\left(e_{1}+y_{r}, e_{2}+\dot{y}_{r}\right)-\gamma_{2}(\cdot)+\ddot{y}_{r}(t)-k_{1} e_{1}\right. \\
& \left.-k_{2} e_{2}\right)
\end{aligned}
$$

substituting it in (12) results in

$$
\begin{aligned}
& \dot{e}_{1}=e_{2}, \\
& \dot{e}_{2}=-k_{1} e_{1}-k_{2} e_{2}, \\
& y_{e}=e_{1} .
\end{aligned}
$$

If $k_{1}$ and $k_{2}$ are positive constants the origin of system (14) is an exponentially stable equilibrium point.

Now $v^{-1}\left(u_{e}\right)$ must be the reference signal for the position $x_{1}$ in (11). Define $x_{\mathrm{re}}=v^{-1}\left(u_{e}\right)$ and new error variables $\varepsilon_{1}=$ $x_{1}-x_{\mathrm{re}}$ and $\varepsilon_{2}=x_{2}-\dot{x}_{\mathrm{re}}$ whose dynamics are given by

$$
\begin{aligned}
\dot{\varepsilon}_{1}= & \varepsilon_{2}, \\
\dot{\varepsilon}_{2}= & f_{1}\left(x_{1}, x_{2}, e_{1}+y_{r}, e_{2}+\dot{y}_{r}\right)+g_{1}\left(x_{1}, x_{2}\right) u+\gamma_{1}(\cdot) \\
& -\ddot{x}_{\text {re }} .
\end{aligned}
$$

A control $u$ that stabilizes the origin of system (15) is

$$
\begin{aligned}
u= & \frac{1}{g_{1}\left(x_{1}, x_{2}\right)}\left(-f_{1}\left(x_{1}, x_{2}, e_{1}+y_{r}, e_{2}+\dot{y}_{r}\right)-\gamma_{1}(\cdot)\right. \\
& \left.+\ddot{x}_{\mathrm{re}}-\alpha_{1} \varepsilon_{1}-\alpha_{2} \varepsilon_{2}-\alpha_{3} \operatorname{sign}\left(\varepsilon_{1}\right)\right) ;
\end{aligned}
$$

substituting it in (15) we have

$$
\begin{aligned}
& \dot{\varepsilon}_{1}=\varepsilon_{2}, \\
& \dot{\varepsilon}_{2}=-\alpha_{1} \varepsilon_{1}-\alpha_{2} \varepsilon_{2}-\alpha_{3} \operatorname{sign}\left(\varepsilon_{1}\right) .
\end{aligned}
$$

If the constants $\alpha_{1}, \alpha_{2}$, and $\alpha_{3}$ are positive the origin of system (17) is an exponentially stable equilibrium point.

It is important to note that, because this section considered that we have the measurement of all terms, it is not necessary to incorporate the term discontinuous in the control (16); however this term is very useful when the implementation is done because it will give robustness to closed-loop system.

3.1. Stability Analysis. To prove the stability of the closed-loop system consider the error system

$$
\begin{aligned}
& \dot{e}_{1}=e_{2}, \\
& \dot{e}_{2}=f_{2}\left(e_{1}+y_{r}, e_{2}+\dot{y}_{r}\right)+g_{2} v\left(x_{1}\right)+\gamma_{2}(\cdot)-\ddot{y}_{r}(t), \\
& \dot{\varepsilon}_{1}=\varepsilon_{2}, \\
& \dot{\varepsilon}_{2}=-\alpha_{1} \varepsilon_{1}-\alpha_{2} \varepsilon_{2}-\alpha_{3} \operatorname{sign}\left(\varepsilon_{1}\right) .
\end{aligned}
$$

The last two equations of (18) form a subsystem uncoupled of the $e_{1}$ and $e_{2}$, regardless of the value of $u_{e}$. Then, if $\alpha_{1}, \alpha_{2}$, and $\alpha_{3}$ are positive constants, the origin of this subsystem is exponentially stable [17].

Because $x_{1}$ converges exponentially to $u_{e}, x_{1}$ can be expressed in the form

$$
\begin{aligned}
x_{1} & =v^{-1}\left(\frac { 1 } { g _ { 2 } } \left(-f_{2}\left(e_{1}+y_{r}, e_{2}+\dot{y}_{r}\right)-\gamma_{2}(\cdot)+\ddot{y}_{r}(t)\right.\right. \\
& \left.\left.-k_{1} e_{1}-k_{2} e_{2}\right)\right)+\Delta(\cdot),
\end{aligned}
$$

where $\Delta(\cdot)$ is the difference between $x_{1}$ and $x_{\text {re }}$ and satisfies

$$
|\Delta(\cdot)| \leq \rho_{0} e^{-\sigma_{0} t}
$$

for some positive constants $\rho_{0}$ and $\sigma_{0}$. Substituting (19) in (18) we have

$$
\begin{aligned}
\dot{e}_{1} & =e_{2}, \\
\dot{e}_{2} & =f_{2}\left(e_{1}+y_{r}, e_{2}+\dot{y}_{r}\right)+\gamma_{2}(\cdot)-\ddot{y}_{r}(t) \\
& +g_{2} v\left(v ^ { - 1 } \left(\frac { 1 } { g _ { 2 } } \left(-f_{2}\left(e_{1}+y_{r}, e_{2}+\dot{y}_{r}\right)-\gamma_{2}(\cdot)\right.\right.\right. \\
& \left.\left.\left.+\ddot{y}_{r}(t)-k_{1} e_{1}-k_{2} e_{2}\right)\right)+\Delta(\cdot)\right), \\
\dot{\varepsilon}_{1} & =\varepsilon_{2}, \\
\dot{\varepsilon}_{2} & =-\alpha_{1} \varepsilon_{1}-\alpha_{2} \varepsilon_{2}-\alpha_{3} \operatorname{sign}\left(\varepsilon_{1}\right) .
\end{aligned}
$$


This system can be rewritten in the form

$$
\begin{aligned}
& \dot{e}_{1}=e_{2}, \\
& \dot{e}_{2}=-k_{1} e_{1}-k_{2} e_{2}+\mu(\cdot), \\
& \dot{\varepsilon}_{1}=\varepsilon_{2}, \\
& \dot{\varepsilon}_{2}=-\alpha_{1} \varepsilon_{1}-\alpha_{2} \varepsilon_{2}-\alpha_{3} \operatorname{sign}\left(\varepsilon_{1}\right),
\end{aligned}
$$

where $\mu(\cdot)$ is a term produced by $\Delta(\cdot)$ that satisfies

$$
|\mu(\cdot)| \leq \rho_{1} e^{-\sigma_{1} t}
$$

for some positive constants $\rho_{1}$ and $\sigma_{1}$. System (22) is a piecewise linear system with a vanishing disturbance; therefore, there exist a set of constants $k_{1}, k_{2}, \alpha_{1}, \alpha_{2}$, and $\alpha_{3}$ such that the origin will be an asymptotically stable equilibrium point in a sufficient large region $\Omega \subset \mathfrak{R}^{4}[17,18]$.

\section{Controller Implementation}

The control given in (13) and (16) cannot be implemented directly because $e_{2}, \varepsilon_{2}, \gamma_{1}(\cdot), \gamma_{2}(\cdot)$, and $\ddot{x}_{\text {re }}$ are not available. In this section we present a method to implement this control input. It is based on discontinuous observers with finite time convergence.

4.1. Estimation of $e_{2}$ and $\gamma_{2}(\cdot)$. To estimate $e_{2}$ and $\gamma_{2}(\cdot)$ we propose a finite time observer for the underactuated part:

$$
\begin{aligned}
& \dot{e}_{1}=e_{2}, \\
& \dot{e}_{2}=f_{2}\left(e_{1}+y_{r}, e_{2}+\dot{y}_{r}\right)+g_{2} v\left(x_{1}\right)+\gamma_{2}(\cdot)-\ddot{y}_{r}(t), \\
& y_{e}=e_{1} .
\end{aligned}
$$

The following observer is based on Levant's exact deriver [19]:

$$
\begin{aligned}
\dot{\hat{e}}_{1}= & z_{1}+c_{1,1}\left|e_{1}-\widehat{e}_{1}\right|^{1 / 2} \operatorname{sign}\left(e_{1}-\widehat{e}_{1}\right), \\
\dot{z}_{1}= & c_{2,1} \operatorname{sign}\left(e_{1}-\widehat{e}_{1}\right), \\
\widehat{y}_{1}= & \widehat{e}_{1}, \\
\dot{\hat{e}}_{2}= & g_{2} v\left(x_{1}\right)-\ddot{y}_{r}(t)+z_{2} \\
& +c_{1,2}\left|z_{1}-\widehat{e}_{2}\right|^{1 / 2} \operatorname{sign}\left(z_{1}-\widehat{e}_{2}\right), \\
\dot{z}_{2}= & c_{2,2} \operatorname{sign}\left(z_{1}-\widehat{e}_{2}\right), \\
\hat{y}_{2}= & \widehat{e}_{2} .
\end{aligned}
$$

To show the stability of the observer define the errors $\epsilon_{1}=$ $e_{1}-\widehat{e}_{1}$ and $\epsilon_{2}=e_{2}-\hat{e}_{2}$, whose dynamics are given by

$$
\begin{aligned}
\dot{\epsilon}_{1}= & e_{2}-z_{1}-c_{1,1}\left|\epsilon_{1}\right|^{1 / 2} \operatorname{sign}\left(\epsilon_{1}\right), \\
\dot{z}_{1}= & c_{2,1} \operatorname{sign}\left(\epsilon_{1}\right), \\
\dot{\epsilon}_{2}= & f_{2}\left(e_{1}+y_{r}, e_{2}+\dot{y}_{r}\right)+\gamma_{2}(\cdot)-z_{2} \\
& \quad-c_{1,2}\left|z_{1}-\widehat{e}_{2}\right|^{1 / 2} \operatorname{sign}\left(z_{1}-\widehat{e}_{2}\right), \\
\dot{z}_{2}= & c_{2,2} \operatorname{sign}\left(z_{1}-\widehat{e}_{2}\right) .
\end{aligned}
$$

Making a change of variables in the first two equations

$$
\begin{aligned}
& v_{1}=\epsilon_{1}, \\
& v_{2}=e_{2}-z_{1},
\end{aligned}
$$

we obtain the subsystem

$$
\begin{aligned}
& \dot{v}_{1}=v_{2}-c_{1,1}\left|v_{1}\right|^{1 / 2} \operatorname{sign}\left(v_{1}\right), \\
& \dot{v}_{2}=\dot{e}_{2}-c_{2,1} \operatorname{sign}\left(v_{1}\right) .
\end{aligned}
$$

If $\left|\dot{e}_{2}\right| \leq \delta_{1}$, where $\delta_{1}$ is a known constant, there exist constants $c_{1,1}$ and $c_{2,1}$ such that the trajectories converge in finite time to $\left(v_{1}=0, v_{2}=0\right),[20]$; therefore

$$
z_{1}=e_{2}
$$

in finite time.

The criteria to choosing the constants $c_{1,1}$ and $c_{2,1}$ are

$$
\begin{aligned}
& c_{1,1}>\sqrt{\frac{2}{c_{2,1}+\delta_{1}}} \frac{\left(c_{2,1}+\delta_{1}\right)(1+p)}{(1-p)}, \\
& c_{2,1}>\delta_{1},
\end{aligned}
$$

where $0<p<1$.

For the last two equations in (26),

$$
\begin{aligned}
\dot{\epsilon}_{2}= & f_{2}\left(e_{1}+y_{r}, e_{2}+\dot{y}_{r}\right)+\gamma_{2}(\cdot)-z_{2} \\
& -c_{1,2}\left|\epsilon_{2}-v_{2}\right|^{1 / 2} \operatorname{sign}\left(\epsilon_{2}-v_{2}\right), \\
\dot{z}_{2}= & c_{2,2} \operatorname{sign}\left(\epsilon_{2}-v_{2}\right) .
\end{aligned}
$$

Making a change of variables

$$
\begin{aligned}
& v_{3}=\epsilon_{2}-v_{2}, \\
& v_{4}=f_{2}\left(e_{1}+y_{r}, e_{2}+\dot{y}_{r}\right)+\gamma_{2}(\cdot)-z_{2}-\dot{v}_{2},
\end{aligned}
$$

the dynamics of these variables are given by

$$
\begin{aligned}
& \dot{v}_{3}=v_{4}-c_{1,2}\left|v_{3}\right|^{1 / 2} \operatorname{sign}\left(v_{3}\right), \\
& \dot{v}_{4}=\dot{f}_{2}\left(e_{1}+y_{r}, e_{2}+\dot{y}_{r}\right)+\dot{\gamma}_{2}(\cdot)-\ddot{v}_{2}-c_{2,2} \operatorname{sign}\left(v_{3}\right) .
\end{aligned}
$$

If $\left|\dot{f}_{2}\left(x_{3}, x_{4}\right)+\dot{\gamma}_{2}(\cdot)-\ddot{v}_{2}\right| \leq \delta_{2}$ there exist constants $c_{1,2}$ and $c_{2,2}$ such that the trajectories converge in finite time to $\left(v_{3}=\right.$ $\left.0, v_{4}=0\right)$; therefore

$$
\begin{aligned}
& 0=\epsilon_{2}-v_{2}, \\
& 0=f_{2}\left(e_{1}+y_{r}, e_{2}+\dot{y}_{r}\right)+\gamma_{2}(\cdot)-z_{2}-\dot{v}_{2} .
\end{aligned}
$$

Considering that for (28) $v_{2}=0$ in finite time, after this time, we estimate the velocity error $e_{2}$ and disturbance terms $f_{2}\left(e_{1}+\right.$ $\left.y_{r}, e_{2}+\dot{y}_{r}\right)+\gamma_{2}(\cdot):$

$$
\begin{aligned}
& \widehat{e}_{2}=e_{2}, \\
& z_{2}=f_{2}\left(e_{1}+y_{r}, e_{2}+\dot{y}_{r}\right)+\gamma_{2}(\cdot) .
\end{aligned}
$$

As we can see $\gamma_{2}(\cdot)$ is estimated through $z_{2}$. 
4.2. Estimation of $\varepsilon_{2}, \gamma_{1}(\cdot)$, and $\ddot{x}_{r e}$. Now we design an observer for system (15):

$$
\begin{aligned}
\dot{\varepsilon}_{1}= & \varepsilon_{2}, \\
\dot{\varepsilon}_{2}= & f_{1}\left(x_{1}, x_{2}, e_{1}+y_{r}, e_{2}+\dot{y}_{r}\right)+g_{1}\left(x_{1}, x_{3}\right) u \\
& +\gamma_{1}(\cdot)-\ddot{x}_{\mathrm{re}}, \\
y_{\varepsilon}= & \varepsilon_{1} .
\end{aligned}
$$

The observer is

$$
\begin{aligned}
\dot{\widehat{\varepsilon}}_{1}= & w_{1}+a_{1,1}\left|\varepsilon_{1}-\widehat{\varepsilon}_{1}\right|^{1 / 2} \operatorname{sign}\left(\varepsilon_{1}-\widehat{\varepsilon}_{1}\right), \\
\dot{w}_{1}= & a_{2,1} \operatorname{sign}\left(\varepsilon_{1}-\widehat{\varepsilon}_{1}\right), \\
\widehat{y}_{1}= & \widehat{\varepsilon}_{1}, \\
\dot{\widehat{\varepsilon}}_{2}= & g_{1}\left(x_{1}, x_{3}\right) u+w_{2} \\
& +a_{1,2}\left|w_{1}-\widehat{\varepsilon}_{2}\right|^{1 / 2} \operatorname{sign}\left(w_{1}-\widehat{\varepsilon}_{2}\right), \\
\dot{w}_{2}= & a_{2,2} \operatorname{sign}\left(w_{1}-\widehat{\varepsilon}_{2}\right), \\
\widehat{y}_{2}= & \widehat{\varepsilon}_{2} .
\end{aligned}
$$

To show the stability of the observer define the errors $\epsilon_{3}=$ $\varepsilon_{1}-\widehat{\varepsilon}_{1}$ and $\epsilon_{4}=\varepsilon_{2}-\widehat{\varepsilon}_{2}$, whose dynamics are given by

$$
\begin{aligned}
\dot{\epsilon}_{3}= & \varepsilon_{2}-w_{1}-a_{1,1}\left|\epsilon_{3}\right|^{1 / 2} \operatorname{sign}\left(\epsilon_{3}\right), \\
\dot{w}_{1}= & a_{2,1} \operatorname{sign}\left(\epsilon_{3}\right), \\
\dot{\epsilon}_{4}= & f_{1}\left(x_{1}, x_{2}, e_{1}+y_{r}, e_{2}+\dot{y}_{r}\right)+\gamma_{1}(\cdot)-\ddot{u}_{e}-w_{2} \\
& \quad-a_{1,2}\left|w_{1}-\widehat{\varepsilon}_{2}\right|^{1 / 2} \operatorname{sign}\left(w_{1}-\widehat{\varepsilon}_{2}\right), \\
\dot{w}_{2}= & a_{2,2} \operatorname{sign}\left(w_{1}-\widehat{\varepsilon}_{2}\right) .
\end{aligned}
$$

Making a change of variables for the first two equations ((38)(39)),

$$
\begin{aligned}
& v_{5}=\epsilon_{3}, \\
& v_{6}=\varepsilon_{2}-w_{1},
\end{aligned}
$$

we obtain the subsystem

$$
\begin{aligned}
& \dot{v}_{5}=v_{6}-a_{1,1}\left|v_{5}\right|^{1 / 2} \operatorname{sign}\left(v_{5}\right), \\
& \dot{v}_{6}=\dot{\varepsilon}_{2}-a_{2,1} \operatorname{sign}\left(v_{5}\right) .
\end{aligned}
$$

According to (30), if $\left|\dot{\varepsilon}_{2}\right| \leq \delta_{3}$ there exist constants $a_{1,1}$ and $a_{2,1}$ such that the trajectories converge in finite time to $\left(v_{5}=\right.$ $\left.0, v_{6}=0\right)[20]$; therefore

$$
w_{1}=\varepsilon_{2},
$$

in finite time.

For the last two equations in ((40)-(41)),

$$
\begin{aligned}
\dot{\epsilon}_{4}= & f_{1}\left(x_{1}, x_{2}, e_{1}+y_{r}, e_{2}+\dot{y}_{r}\right)+\gamma_{1}(\cdot)-\ddot{x}_{\mathrm{re}}-w_{2} \\
& -a_{1,2}\left|\epsilon_{4}-v_{6}\right|^{1 / 2} \operatorname{sign}\left(\epsilon_{4}-v_{6}\right), \\
\dot{w}_{2}= & a_{2,2} \operatorname{sign}\left(\epsilon_{4}-v_{6}\right) .
\end{aligned}
$$

Making a change of variables

$$
\begin{aligned}
v_{7}= & \epsilon_{4}-v_{6}, \\
v_{8}= & f_{1}\left(x_{1}, x_{2}, e_{1}+y_{r}, e_{2}+\dot{y}_{r}\right)+\gamma_{1}(\cdot)-\ddot{x}_{\mathrm{re}}-\dot{v}_{6} \\
& -w_{2},
\end{aligned}
$$

the dynamics of these variables are given by

$$
\begin{aligned}
\dot{v}_{7}= & v_{8}-a_{1,2}\left|v_{7}\right|^{1 / 2} \operatorname{sign}\left(v_{7}\right), \\
\dot{v}_{8}= & \dot{f}_{1}\left(x_{1}, x_{2}, x_{3}, x_{4}\right)+\dot{\gamma}_{1}(\cdot)-\ddot{x}_{\mathrm{re}}-\ddot{v}_{6} \\
& -a_{2,2} \operatorname{sign}\left(v_{7}\right) .
\end{aligned}
$$

If $\left|\dot{f}_{1}\left(x_{1}, x_{2}, e_{1}+y_{r}, e_{2}+\dot{y}_{r}\right)+\dot{\gamma}_{1}(\cdot)-\ddot{x}_{\mathrm{re}}-\ddot{v}_{6}\right| \leq \delta_{4}$ there exist constants $a_{1,2}$ and $a_{2,2}$ such that the trajectories converge in finite time to $\left(v_{7}=0, v_{8}=0\right)[20]$; therefore

$$
\begin{aligned}
0= & \epsilon_{4}-v_{6}, \\
0= & f_{1}\left(x_{1}, x_{2}, e_{1}+y_{r}, e_{2}+\dot{y}_{r}\right)+\gamma_{1}(\cdot)-\ddot{x}_{\mathrm{re}}-\dot{v}_{6} \\
& -w_{2} .
\end{aligned}
$$

Considering that for (43) $v_{6}=0$ in finite time, after a finite time, we have

$$
\begin{aligned}
\widehat{\varepsilon}_{2} & =\varepsilon_{2} \\
w_{2} & =f_{1}\left(x_{1}, x_{2}, e_{1}+y_{r}, e_{2}+\dot{y}_{r}\right)+\gamma_{1}(\cdot)-\ddot{x}_{\mathrm{re}}
\end{aligned}
$$

Now, the control inputs (13) and (16) may be implemented in the following form:

$$
\begin{aligned}
x_{\mathrm{re}} & \approx \frac{1}{g_{2}}\left(-z_{2}+\ddot{y}_{r}(t)-k_{1} e_{1}-k_{2} \widehat{e}_{2}\right), \\
u & \approx \frac{1}{g_{1}\left(x_{1}, x_{3}\right)}\left(-w_{2}-\alpha_{1} \varepsilon_{1}-\alpha_{2} \widehat{\varepsilon}_{2}-\alpha_{3} \operatorname{sign}\left(\varepsilon_{1}\right)\right) .
\end{aligned}
$$

The implementation of the controller must be in several stages. First we have to apply a signal $u$, in open loop, such that the behavior of the system will be bounded; in this way the observers can estimate the state and disturbances in finite time. After this time, the control signals (50) can be implemented and then close the control loop.

\section{Control System Performance}

This section shows the performance of the control system through numerical simulations and experimental results; the control systems are a ball and beam system and a springmass-damper mechanism.

5.1. Control of a Ball and Beam System. Consider the ball and beam system shown in Figure 1; its model is given by

$$
\begin{array}{r}
\left(J+m x^{2}\right) \ddot{\alpha}+2 m x \dot{\alpha} \dot{x}-(m g x) \cos (\alpha)+\delta_{1} \dot{\alpha}=u, \\
\frac{7}{5} \ddot{x}-x \dot{\alpha}^{2}-g \sin (\alpha)+\delta_{2} \dot{x}=0,
\end{array}
$$




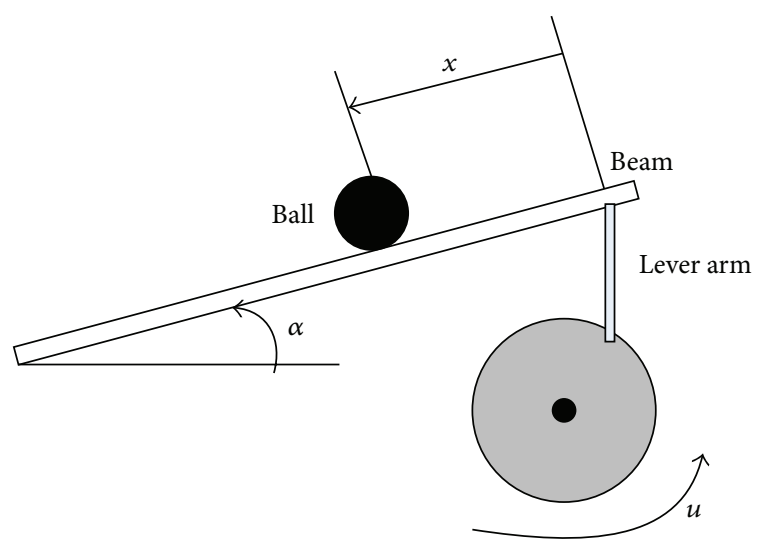

FIGURE 1: Ball and beam mechanical system.

where $x$ is the position of the ball, $\alpha$ is the angle of the frame, $J=0.032 \mathrm{~kg} \cdot \mathrm{m}^{2}$ is the moment of inertia of the beam, $m=$ $0.06 \mathrm{~kg}$ is the mass of the ball, $\delta_{1}$ and $\delta_{2}$ are viscous friction coefficients, and $g=9.8 \mathrm{~m} / \mathrm{seg}^{2}$ is the gravitational force. Defining the state variables as $x_{1}=\alpha, x_{2}=\dot{\alpha}, x_{3}=x$, and $x_{4}=\dot{x}$ and substituting the values of the constants we have the model

$$
\begin{aligned}
\ddot{\alpha}= & \frac{m x \dot{\alpha} \dot{x}}{\left(J+m x^{2}\right)}+\frac{(m g x) \cos (\alpha)}{\left(J+m x^{2}\right)}-\frac{\delta_{1}}{\left(J+m x^{2}\right)} \dot{\alpha} \\
& +\frac{u}{\left(J+m x^{2}\right)}, \\
\ddot{x}= & \frac{5}{7} x \dot{\alpha}^{2}-\frac{5}{7} \delta_{2} \dot{x}+\frac{5}{7} g \sin (\alpha) .
\end{aligned}
$$

A state variable representation is as follows:

$$
\begin{aligned}
\dot{x}_{1}= & x_{2}, \\
\dot{x}_{2}= & \frac{m x_{3} x_{2} x_{4}}{\left(J+m x_{3}^{2}\right)}+\frac{\left(m g x_{3}\right) \cos \left(x_{1}\right)}{\left(J+m x_{3}^{2}\right)}-\frac{\delta_{1}}{\left(J+m x^{2}\right)} x_{2} \\
& +\frac{u}{\left(J+m x_{3}^{2}\right)}, \\
\dot{x}_{3}= & x_{4}, \\
\dot{x}_{4}= & \frac{5}{7} x_{3} x_{2}^{2}-\frac{5}{7} \delta_{2} x_{4}+\frac{5}{7} g \sin \left(x_{1}\right), \\
y_{1}= & x_{1}, \\
y_{2}= & x_{3} .
\end{aligned}
$$

In this example, without loss of generality, the model is free from uncertainties and external disturbances. It is important to note that the variable $x_{1}$ is the argument of the sine function, so the control will have a bounded amplitude.

The control objective is that the ball position $x_{3}$ tracks the reference signal $y_{r}(t)$. Define the error variables $e_{1}=x_{3}-y_{r}(t)$ and $e_{2}=x_{4}-\dot{y}_{r}(t)$ and the auxiliary control $u_{e}=\sin \left(x_{1}\right)$ to obtain the following system:

$$
\begin{aligned}
\dot{x}_{1}= & x_{2}, \\
\dot{x}_{2}= & \frac{m x_{3} x_{2} x_{4}}{\left(J+m x_{3}^{2}\right)}+\frac{\left(m g x_{3}\right) \cos \left(x_{1}\right)}{\left(J+m x_{3}^{2}\right)}-\frac{\delta_{1}}{\left(J+m x^{2}\right)} x_{2} \\
& +\frac{u}{\left(J+m x_{3}^{2}\right)}, \\
\dot{e}_{1}= & e_{2}, \\
\dot{e}_{2}= & \frac{5}{7} x_{3} x_{2}^{2}-\frac{5}{7} \delta_{2} x_{4}-\ddot{y}_{r}(t)+\frac{5}{7} g u_{e}, \\
y_{1}= & x_{1}, \\
y_{e}= & e_{1},
\end{aligned}
$$

where $u_{e}$ only may take values in the $[-1,1]$ interval. (54) is

The ideal controller $u_{e}$ to stabilize the origin of system

$$
u_{e}=\frac{7}{5 g}\left(-\frac{5}{7} x_{3} x_{2}^{2}+\frac{5}{7} \delta_{2} x_{4}+\ddot{y}_{r}(t)-k_{1} e_{1}-k_{2} e_{2}\right),
$$

where $k_{1}=30$ and $k_{2}=10$. Thus, the reference signal for $x_{1}$ is

$$
\begin{aligned}
x_{\mathrm{re}} & =\arcsin \left(\frac { 7 } { 5 g } \left(-\frac{5}{7} x_{3} x_{2}^{2}+\frac{5}{7} \delta_{2} x_{4}+\ddot{y}_{r}(t)-k_{1} e_{1}\right.\right. \\
& \left.\left.-k_{2} e_{2}\right)\right) .
\end{aligned}
$$

Define new error variables $\varepsilon_{1}=x_{1}-x_{\text {re }}$ and $\varepsilon_{2}=x_{2}-\dot{x}_{\text {re }}$ whose dynamics are given by

$$
\begin{aligned}
\dot{\varepsilon}_{1}= & \varepsilon_{2}, \\
\dot{\varepsilon}_{2}= & \frac{m x_{3} x_{2} x_{4}}{\left(J+m x_{3}^{2}\right)}+\frac{\left(m g x_{3}\right) \cos \left(x_{1}\right)}{\left(J+m x_{3}^{2}\right)}-\frac{\delta_{1}}{\left(J+m x^{2}\right)} x_{2} \\
& -\ddot{x}_{\mathrm{re}}+\frac{u}{\left(J+m x_{3}^{2}\right)} .
\end{aligned}
$$

Then, an ideal control to stabilize the origin of system (57) is

$$
\begin{aligned}
u & =\left(J+m x_{3}^{2}\right)\left(-\frac{m x_{3} x_{2} x_{4}}{\left(J+m x_{3}^{2}\right)}-\frac{\left(m g x_{3}\right) \cos \left(x_{1}\right)}{\left(J+m x_{3}^{2}\right)}\right. \\
& \left.+\frac{\delta_{1}}{\left(J+m x^{2}\right)} x_{2}+\ddot{x}_{\mathrm{re}}+u_{o}\right), \\
u_{o} & =-\sigma_{1} \varepsilon_{1}-\sigma_{2} \varepsilon_{2}-\sigma_{3} \operatorname{sign}\left(\varepsilon_{1}\right),
\end{aligned}
$$

where $\sigma_{1}=40, \sigma_{2}=10$, and $\sigma_{3}=0.7$. Figure 2 shows the results when the reference $y_{r}$ takes different constant values; this is the case of regulation. Steady state error is practically zero and the control signal takes values suitable for a possible implementation. 

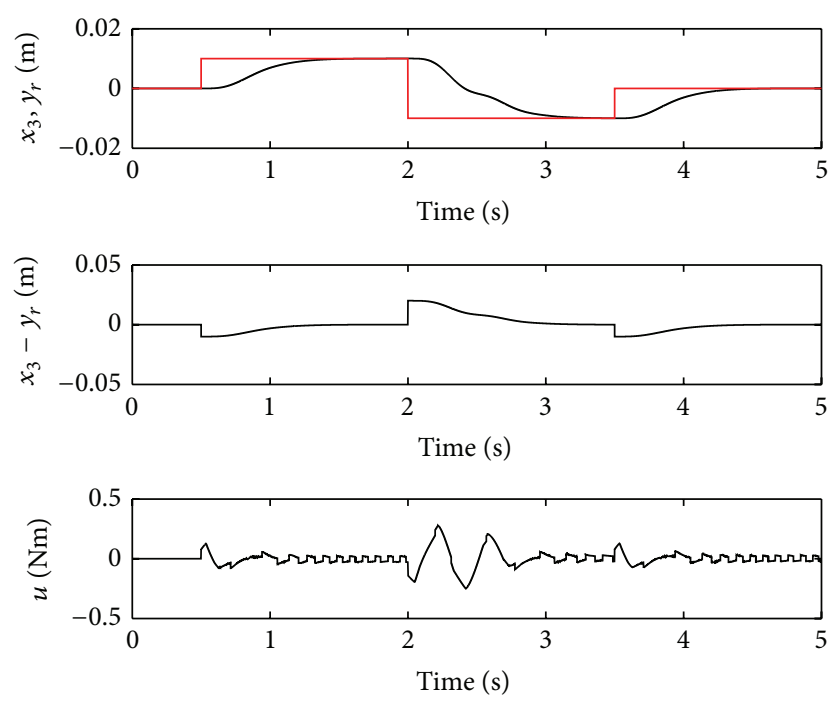

FIGURE 2: Simulation results with the ball and beam system; performance for the regulation problem.

The same situation occurs when the reference is a timevarying signal. Figure 3 shows that the output signal $x_{3}$ of the nonactuated link converges to a time-varying signal with an almost zero steady-state error and a control signal with adequate performance for experimental implementation.

5.2. Control of a Mass-Spring-Damper System. Consider the 2DOF underactuated mass-spring-damper mechanical system shown in Figure 4, with the model

$$
\begin{aligned}
m_{1} \ddot{x}= & -k_{1} x-\delta_{1} \dot{x}+(z-x) k_{2}+(\dot{z}-\dot{x}) \delta_{2}+k_{m} u \\
& +\gamma_{1}(\cdot), \\
m_{2} \ddot{z}= & -(z-x) k_{2}-(\dot{z}-\dot{x}) \delta_{2}+\gamma_{2}(\cdot),
\end{aligned}
$$

where $x, \dot{x}$, and $\ddot{x}$ are the position, velocity, and acceleration of the first mass, $z, \dot{z}$, and $\ddot{z}$ are the position, velocity, and acceleration of the second mass, $u$ is the control input, and $\gamma_{1}(\cdot)$ and $\gamma_{2}(\cdot)$ are disturbances that include terms produced by parameter uncertainties.

The nominal parameters are $k_{1}=k_{2}=189.65 \mathrm{~N} / \mathrm{m}$, $\delta_{1}=10.54 \mathrm{~kg} / \mathrm{sec}, \delta_{2}=1.19 \mathrm{~kg} / \mathrm{sec}, m_{1}=0.77 \mathrm{~kg}, m_{2}=$ $0.60 \mathrm{~kg}$, and $k_{m}=2.85 \mathrm{~N} / \mathrm{V}$; these are the nominal parameter values for the mass-spring-damper system manufactured by Educational Control Products Inc. A state representation of system (59) is

$$
\begin{aligned}
\dot{x}_{1}= & x_{2}, \\
\dot{x}_{2}= & -491.74 x_{1}-15.23 x_{2}+10245.87 x_{3}+1.55 x_{4} \\
& +3.69 u+\gamma_{1}(\cdot),
\end{aligned}
$$
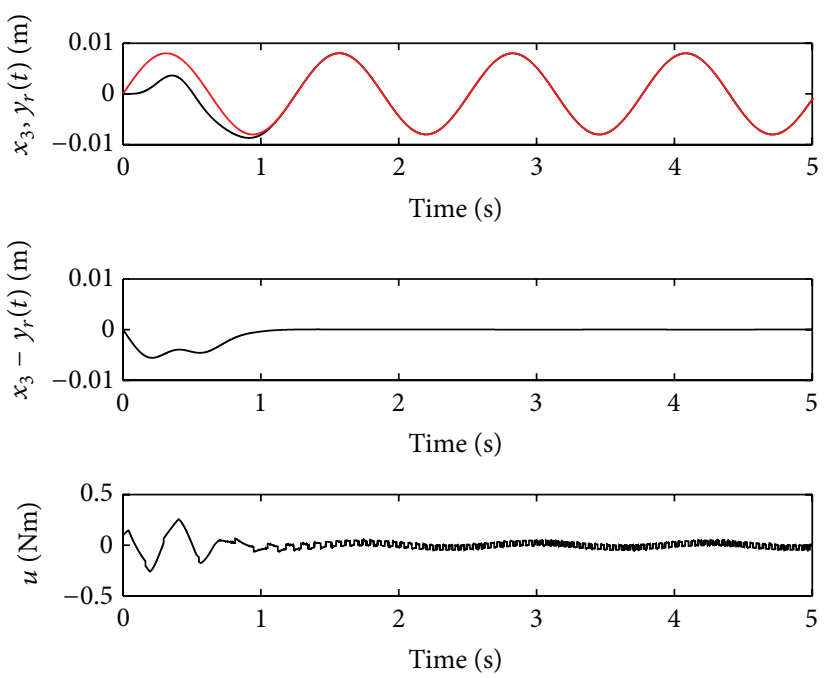

FIGURE 3: Simulation results with the ball and beam system; performance for the tracking problem.

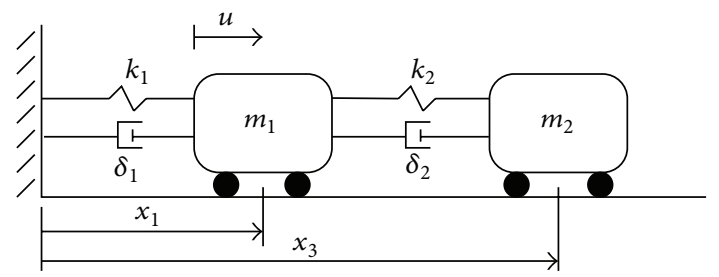

FIGURE 4: Underactuated mass-spring-damper mechanical systems.

$$
\begin{aligned}
& \dot{x}_{3}=x_{4}, \\
& \dot{x}_{4}=312.72 x_{1}+1.97 x_{2}-312.72 x_{3}-1.97 x_{4}+\gamma_{2}(\cdot), \\
& y_{1}=x_{1}, \\
& y_{2}=x_{3} .
\end{aligned}
$$

The control objective is

$$
\lim _{t \rightarrow \infty}\left|x_{3}-y_{r}(t)\right|=0
$$

with a bounded behavior in $x_{1}, x_{2}$, and $x_{4}$. by

Define the error $e_{1}=x_{3}-y_{r}(t)$ whose dynamics are given

$$
\begin{aligned}
\dot{e}_{1}= & e_{2}, \\
\dot{e}_{2}= & -312.72 e_{1}-1.97 e_{2}-312.72 y_{r}(t)-1.97 \dot{y}_{r}(t) \\
& \quad-\ddot{y}_{r}(t)+1.97 x_{2}+\gamma_{2}(\cdot)+312.72 x_{1}, \\
y_{e}= & e_{1}, \\
\dot{x}_{1}= & x_{2},
\end{aligned}
$$




$$
\begin{aligned}
\dot{x}_{2}= & -491.74 x_{1}-15.23 x_{2}+10245.87 x_{3}+1.55 x_{4} \\
& +3.69 \tau+\gamma_{1}(\cdot) \\
y_{1}= & x_{1} .
\end{aligned}
$$

In this case the signal $u_{e}=x_{\mathrm{re}}$ and is given by

$$
\begin{aligned}
x_{\mathrm{re}}= & 3.19 \\
& \times 10^{-3}\left(-c_{1} e_{1}-c_{2} e_{2}-1.97 x_{2}-\gamma_{2}(\cdot)+\Theta\right),
\end{aligned}
$$

where $c_{1}=c_{2}=10$ and $\Theta=312.72 y_{r}(t)+1.97 \dot{y}_{r}(t)+\ddot{y}_{r}(t)$.

To design the control $u$ define the errors

$$
\begin{aligned}
& \varepsilon_{1}=x_{1}-x_{\mathrm{re}}, \\
& \varepsilon_{2}=x_{2}-\dot{x}_{\mathrm{re}},
\end{aligned}
$$

with dynamics given by

$$
\begin{aligned}
\dot{\varepsilon}_{1}= & \varepsilon_{2} \\
\dot{\varepsilon}_{2}= & -491.74 x_{1}-15.23 x_{2}+10245.87 x_{3}+1.55 x_{4} \\
& +3.69 u+\gamma_{1}(\cdot)-\ddot{x}_{\mathrm{re}}
\end{aligned}
$$

and the control input for this subsystem is

$$
\begin{aligned}
u= & 0.270\left(491.74 x_{1}+15.23 x_{2}-10245.87 x_{3}\right. \\
& \left.-1.55 x_{4}-\gamma_{1}(\cdot)+\ddot{x}_{\mathrm{re}}+u_{o}\right), \\
u_{o} & =-\alpha_{1} \varepsilon_{1}-\alpha_{2} \varepsilon_{2}-\alpha_{3} \operatorname{sign}\left(\varepsilon_{1}\right) .
\end{aligned}
$$
$\gamma_{2}(\cdot)$ is

The observer used to estimate the unknown signals $e_{2}$ and

$$
\begin{aligned}
\dot{\hat{e}}_{1}= & z_{1}+c_{1,1}\left|e_{1}-\widehat{e}_{1}\right|^{1 / 2} \operatorname{sign}\left(e_{1}-\widehat{e}_{1}\right), \\
\dot{z}_{1}= & c_{2,1} \operatorname{sign}\left(e_{1}-\widehat{e}_{1}\right), \\
\hat{y}_{1}= & \widehat{e}_{1}, \\
\dot{\hat{e}}_{2}= & -312.72 e_{1}-1.97 e_{2}-312.72 y_{r}(t)-1.97 \dot{y}_{r}(t) \\
& -\ddot{y}_{r}(t)+312.72 x_{1}+z_{2} \\
& +c_{1,2}\left|z_{1}-\widehat{e}_{2}\right|^{1 / 2} \operatorname{sign}\left(z_{1}-\widehat{e}_{2}\right), \\
\dot{z}_{2}= & c_{2,2} \operatorname{sign}\left(z_{1}-\widehat{e}_{2}\right), \\
\widehat{y}_{2}= & \widehat{e}_{2},
\end{aligned}
$$

where $z_{1} \approx e_{2}$ and $z_{2} \approx \gamma_{2}(\cdot)$.
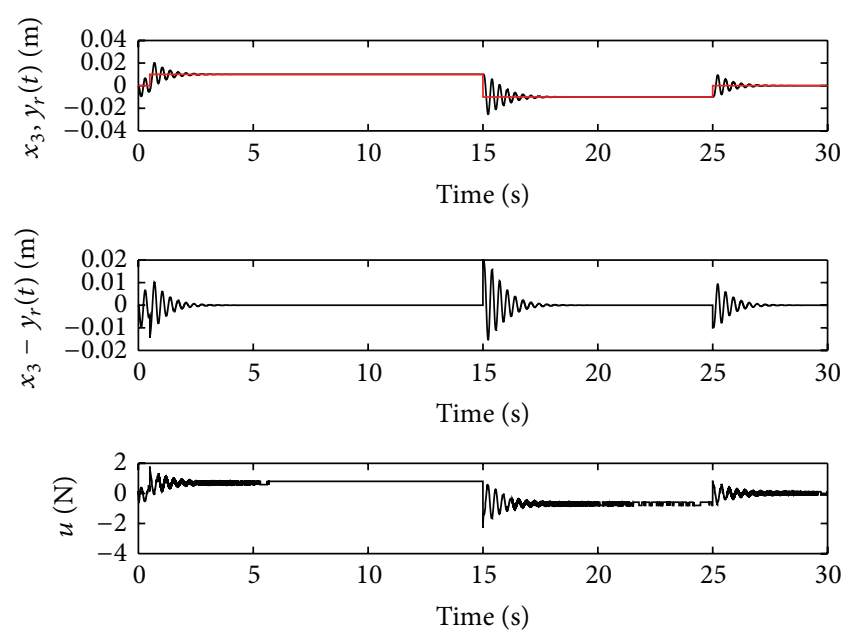

FIgURE 5: Simulation results with the mass-spring-damper system; performance for the regulation problem.

The observer to estimate $\varepsilon_{2}, \gamma_{1}(\cdot)$, and $\ddot{x}_{\text {re }}$ is

$$
\begin{aligned}
\dot{\widehat{\varepsilon}}_{1}= & w_{1}+a_{1,1}\left|\varepsilon_{1}-\widehat{\varepsilon}_{1}\right|^{1 / 2} \operatorname{sign}\left(\varepsilon_{1}-\widehat{\varepsilon}_{1}\right), \\
\dot{w}_{1}= & a_{2,1} \operatorname{sign}\left(\varepsilon_{1}-\widehat{\varepsilon}_{1}\right), \\
\widehat{y}_{1}= & \widehat{\varepsilon}_{1}, \\
\dot{\widehat{\varepsilon}}_{2}= & -491.74 x_{1}+10245.87 x_{3}+3.69 u+w_{2} \\
& +a_{1,2}\left|w_{1}-\widehat{\varepsilon}_{2}\right|^{1 / 2} \operatorname{sign}\left(w_{1}-\widehat{\varepsilon}_{2}\right), \\
\dot{w}_{2}= & a_{2,2} \operatorname{sign}\left(w_{1}-\widehat{\varepsilon}_{2}\right), \\
\widehat{y}_{2}= & \widehat{\varepsilon}_{2},
\end{aligned}
$$

where $w_{1} \approx \varepsilon_{2}$ and $w_{2} \approx-491.74 x_{1}-15.23 x_{2}+10245.87 x_{3}+$ $1.55 x_{4}+\gamma_{1}(\cdot)-\ddot{x}_{\text {re }}$.

The control inputs (13) and (16) may be implemented in the following form:

$$
\begin{aligned}
x_{\mathrm{re}} & \approx \frac{1}{g_{2}}\left(-z_{2}+\ddot{y}_{r}(t)-k_{1} e_{1}-k_{2} \widehat{e}_{2}\right), \\
u & \approx \frac{1}{g_{1}\left(x_{1}, x_{3}\right)}\left(-w_{2}-\alpha_{1} \varepsilon_{1}-\alpha_{2} \widehat{\varepsilon}_{2}-\alpha_{3} \operatorname{sign}\left(\varepsilon_{1}\right)\right),
\end{aligned}
$$

where $k_{1}=10, k_{2}=1, \alpha_{1}=20, \alpha_{2}=20$, and $\alpha_{3}=0.4$.

5.2.1. Numerical Results. Figure 5 shows the results when the reference $y_{r}$, red line, takes different constant values; this is the case of regulation. As can be seen, the output signal $x_{3}$ (black line) reaches asymptotically the reference after a short transient. The steady state error $x_{3}-y_{r}(t)$ is practically zero and the control signal $u$ takes values suitable for a possible implementation.

The same situation occurs when the reference is a timevarying signal. Figure 6 shows that the output signal $x_{3}$ (black line) of the unactuated link converges to a time-varying signal 

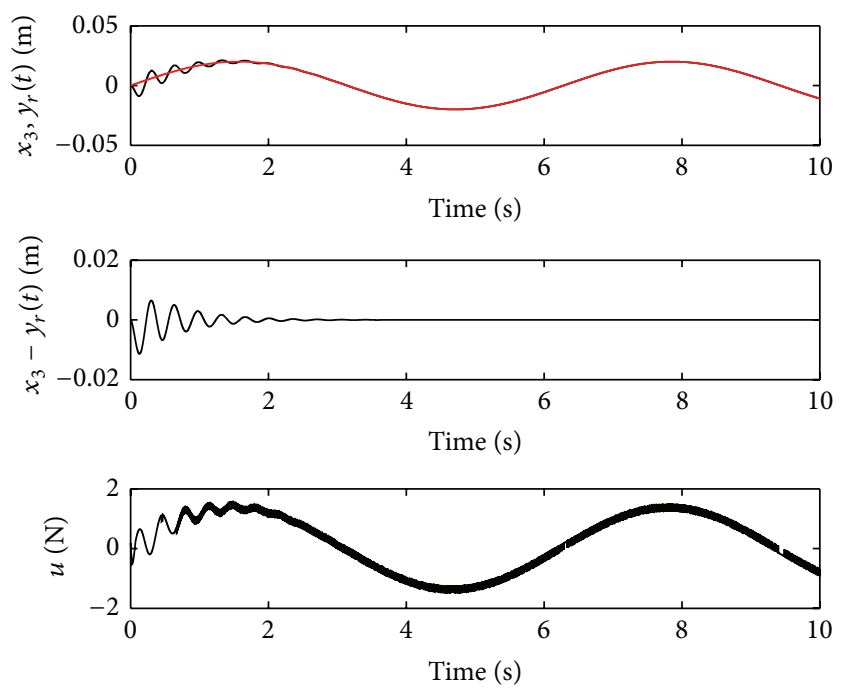

Figure 6: Simulation results with the mass-spring-damper system; performance for the tracking problem.
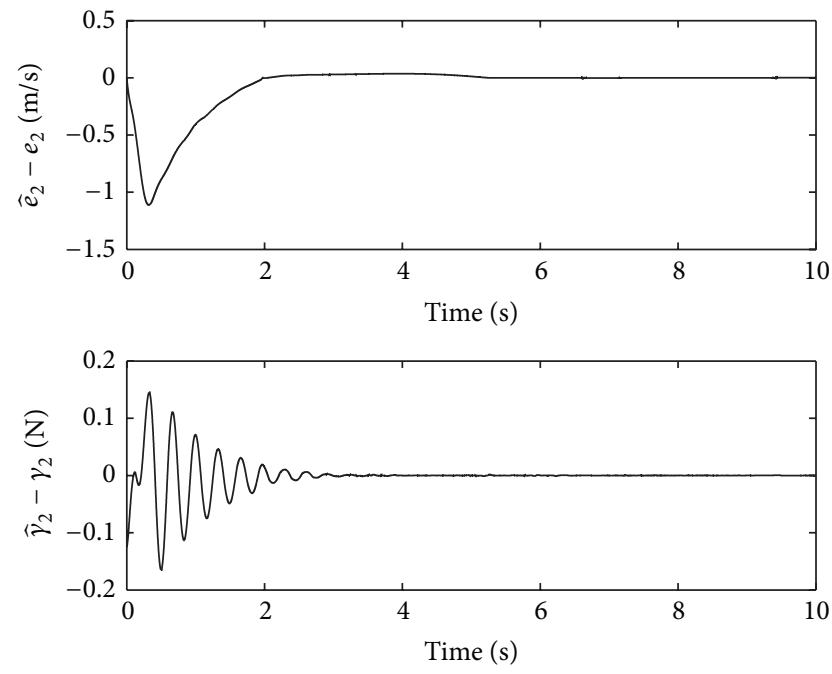

FIGURE 7: Estimation errors of $e_{2}$ and $\gamma_{2}(\cdot)$.

$y_{r}(t)$ (red line) with an almost zero steady-state error, $x_{3}-$ $y_{r}(t)$, and a control signal $u$ with good characteristics for experimental implementation.

For this last case, we analyze the behavior of the state observers. Figure 7 shows the behavior of the errors $\widehat{e}_{2}-e_{2}$ and $\hat{\gamma}_{2}(\cdot)-\gamma_{2}(\cdot)$; these errors converge to zero in few seconds.

For the observer that estimates $\varepsilon_{2}$ and the term that includes $\gamma_{1}(\cdot)$ and $\ddot{x}_{\text {re }}$, it is not possible to compare the actual values with the estimate values. Therefore we check the behavior of the errors $\varepsilon_{1}-\widehat{\varepsilon}_{1}$ and $\varepsilon_{2}-\widehat{\varepsilon}_{2}$; as these errors go to zero, as we can see in Figure 8, the estimation of $\varepsilon_{2}$ and the term that includes $\gamma_{1}(\cdot)$ and $\ddot{x}_{\text {re }}$ is correct.

5.2.2. Experimental Results. The proposed controller is applied to a spring mass damping system manufactured by Educational Control Products Inc., shown in Figure 9. In this
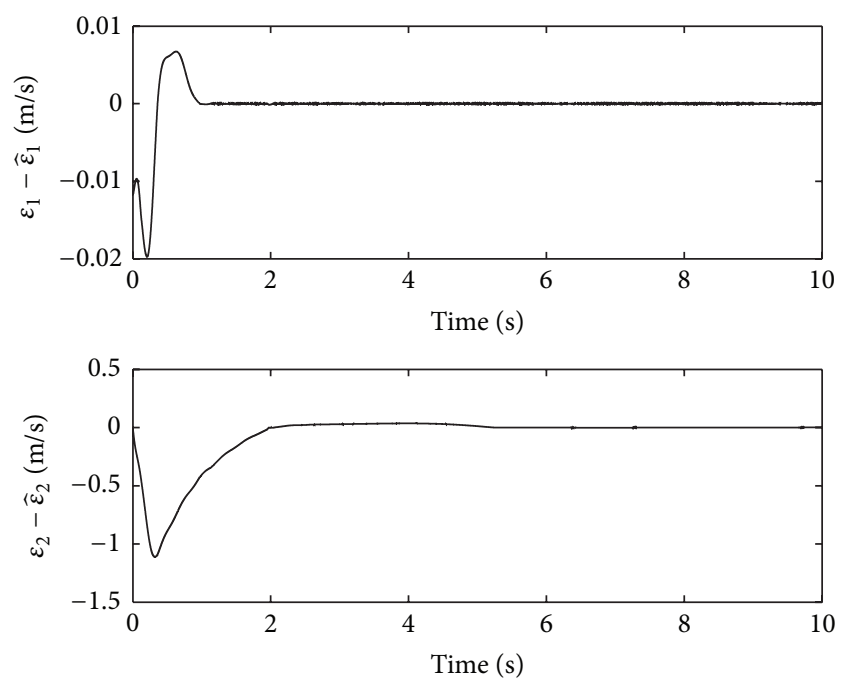

Figure 8: Behavior of the internal errors of the observer that estimates $\varepsilon_{2}, \gamma_{1}(\cdot)$, and $\ddot{x}_{\mathrm{re}}$.

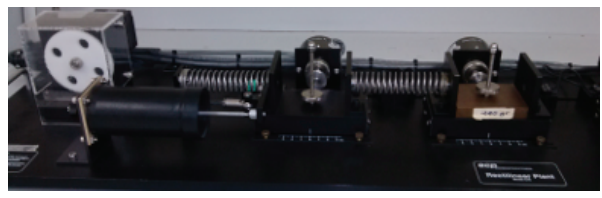

FIGURE 9: Mass damper spring system used in the experiments.

experiment it is assumed that the plant has the same parameters as those considered in the numerical simulation of the previous section and did not conduct a rigorous procedure to estimate the parameters of the real plant, creating a significant challenge to the controller. This situation was resolved by tuning the parameters of each observer, the internal control signal $u_{e}$, and the total control $u$; where $k_{1}=20, k_{2}=2$, $\alpha_{1}=20, \alpha_{2}=5$, and $\alpha_{3}=0.4$.

Experimental results are shown with a reference signal $y_{r}$ with constant values at different times; that is, the control objective is regulation. The results are shown in Figure 10, where we can see that the transient takes about one second; the amplitude of the steady-state error, $x_{3}-y_{r}$, has a maximum of $8 \times 10^{-6}$ meters and the control signal $u$ takes values which are in the permissible range of the control system, \pm 3 volts.

In the second experiment we apply a time varying signal; a sine function, that is, the control target, is tracking. The results are shown in Figure 11, where we can see that the transient takes about 5.8 seconds with an initial error of about 0.01 meters, the steady-state error amplitude, $x_{3}-y_{r}(t)$, has a maximum of $\pm 3 \times 10^{-4}$ meters, and the control signal takes values which are in the permissible range control system, as in the previous case, between \pm 3 volts.

\section{Conclusions}

The control strategy proposed formally guarantees the control objective, either regulation or trajectory tracking, and at 

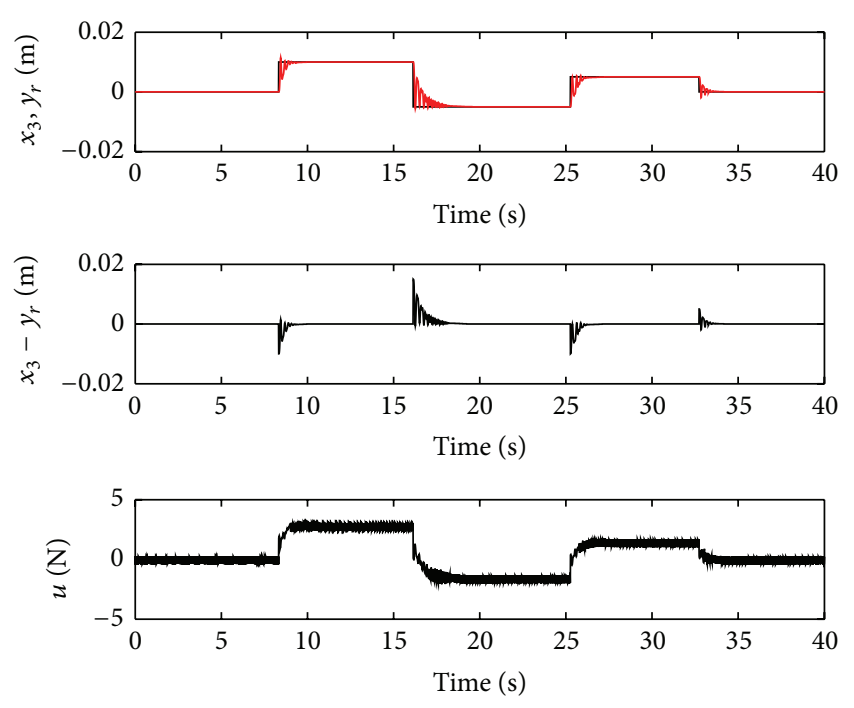

FIGURE 10: Experimental results and the regulation case.
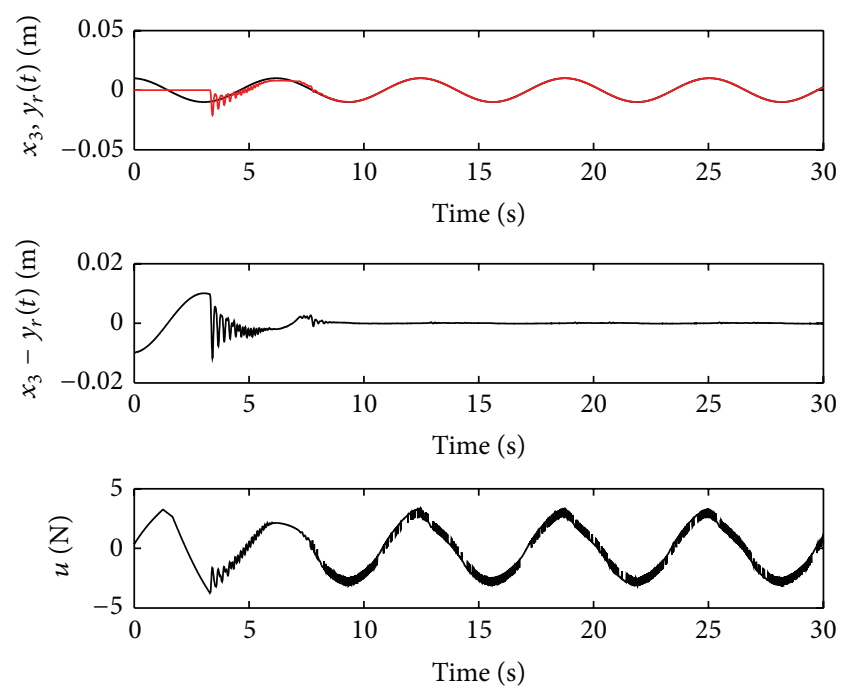

FIGURE 11: Experimental results and tracking case.

the same time establishes a strategy for its implementation considering partial measurement of the state variables and parametric uncertainties. Although stability is not global, the subspace that can ensure stability can be made as large as needed in practice. Some of its limitations are the number of parameters to adjust, both in the observers and in the controller, and the need to use a real-time platform to implement the controller to ensure a sample time less than or equal to one millisecond, and thus the actual sliding mode, produced by discontinuous terms, enough approaches the ideal sliding mode.

\section{Conflict of Interests}

The authors declare that there is no conflict of interests regarding the publication of this paper.

\section{References}

[1] N. Adhikary and C. Mahanta, "Integral backstepping sliding mode control for underactuated systems: swing-up and stabilization of the Cart-Pendulum System," ISA Transactions, vol. 52, no. 6, pp. 870-880, 2013.

[2] S. Andary, A. Chemori, M. Benoit, and J. Sallantin, "A dual model-free control of underactuated mechanical systems, application to the inertia wheel inverted pendulum," in Proceedings of the American Control Conference (ACC '12), pp. 1029-1034, June 2012.

[3] M. Bettayeb, C. Boussalem, R. Mansouri, and U. M. AlSaggaf, "Stabilization of an inverted pendulum-cart system by fractional PI-state feedback," ISA Transactions, vol. 53, no. 2, pp. 508-516, 2014.

[4] L. Xu, Q. Hu, and G. Ma, "Output feedback stabilization control for underactuated mechanical systems," in Proceedings of the 31st Chinese Control Conference (CCC '12), pp. 4267-4272, IEEE, July 2012.

[5] F. Mnif, "VSS control for a class of underactuated mechanical systems," International Journal of computational Cognition, vol. 3, no. 2, 2005.

[6] P. Morin and C. Samson, "Control of underactuated mechanical systems by the transverse function approach," in Proceedings of the 44th IEEE Conference on Decision and Control, and the European Control Conference (CDC-ECC '05), pp. 7508-7513, IEEE, December 2005.

[7] M. S. Park, D. Chwa, and S. K. Hong, "Decoupling control of a class of underactuated mechanical systems based on sliding mode control," in Proceedings of the International Joint Conference (SICE-ICASE '06), pp. 806-810, IEEE, 2006.

[8] M. Reyhanoglu, A. van der Schaft, N. H. McClamroch, and I. Kolmanovsky, "Dynamics and control of a class of underactuated mechanical systems," IEEE Transactions on Automatic Control, vol. 44, no. 9, pp. 1663-1671, 1999.

[9] V. Sankaranarayanan and A. D. Mahindrakar, "Control of a class of underactuated mechanical systems using sliding modes," IEEE Transactions on Robotics, vol. 25, no. 2, pp. 459-467, 2009.

[10] W. Wang, J. Yi, D. Zhao, and X. Liu, "Double layer sliding mode control for second-order underactuated mechanical systems," in Proceedings of the IEEE IRS/RSJ International Conference on Intelligent Robots and Systems (IROS '05), pp. 295-300, IEEE, August 2005.

[11] Y.-F. Chen and A.-C. Huang, "Controller design for a class of underactuated mechanical systems," IET Control Theory \& Applications, vol. 6, no. 1, pp. 103-110, 2012.

[12] S. Rudra, R. K. Barai, M. Maitra et al., "Global stabilization of a flat underactuated inertia wheel: a block backstepping approach," in Proceedings of the 3rd International Conference on Computer Communication and Informatics (ICCCI '13), pp. 1-4, IEEE, January 2013.

[13] J.-X. Xu, Z.-Q. Guo, and T. H. Lee, "Sliding mode controller design for underactuated systems," in Proceedings of the 12th International Workshop on Variable Structure Systems (VSS '12), pp. 385-390, January 2012.

[14] R. M. T. Raja Ismail, D. T. Nguyen, and Q. P. Ha, “Observerbased trajectory tracking for a class of underactuated Lagrangian systems using higher-order sliding modes," in Proceedings of the IEEE International Conference on Automation Science and Engineering: Green Automation Toward a Sustainable Society (CASE '12), pp. 1204-1209, August 2012. 
[15] C.-C. Cheng, K.-S. Yang, and J.-H. Yang, "Robust finite time controller design for second order nonlinear underactuated mechanical systems," Transactions of the Canadian Society for Mechanical Engineering, vol. 37, no. 3, pp. 549-557, 2013.

[16] P. K. Khosla and T. Kanade, "Parameter identification of robot dynamics," in Proceedings of the 24th IEEE Conference on Decision and Control, pp. 1754-1760, IEEE, Fort Lauderdale, Fla, USA, December 1985.

[17] D. I. R. Almeida, J. Alvarez, and L. Fridman, "Robust observation and identification of $n$ DOF Lagrangian systems," International Journal of Robust and Nonlinear Control, vol. 17, no. 9, pp. 842-861, 2007.

[18] H. K. Khalil, Nonlinear Systems, vol. 3, Prentice Hall, Upper Saddle River, NJ, USA, 2002.

[19] A. Levant, "Robust exact differentiation via sliding mode technique," Automatica, vol. 34, no. 3, pp. 379-384, 1998.

[20] J. Davila, L. Fridman, and A. Levant, "Second-order slidingmode observer for mechanical systems," IEEE Transactions on Automatic Control, vol. 50, no. 11, pp. 1785-1789, 2005. 


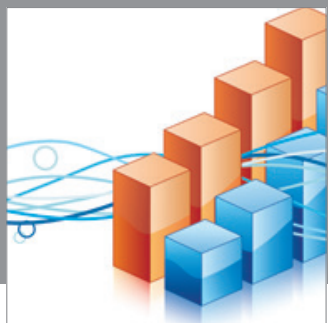

Advances in

Operations Research

mansans

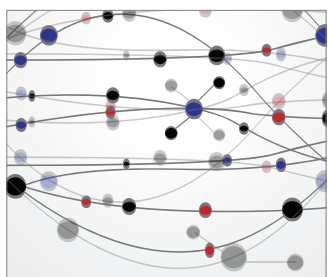

The Scientific World Journal
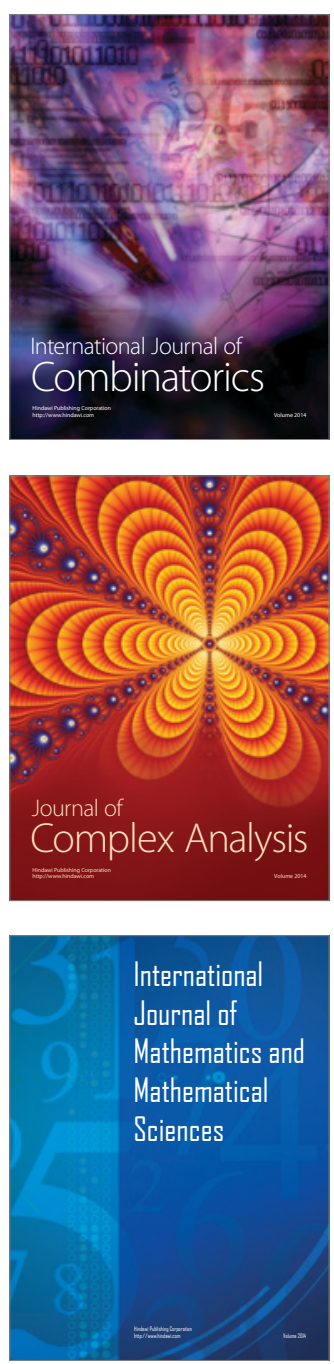
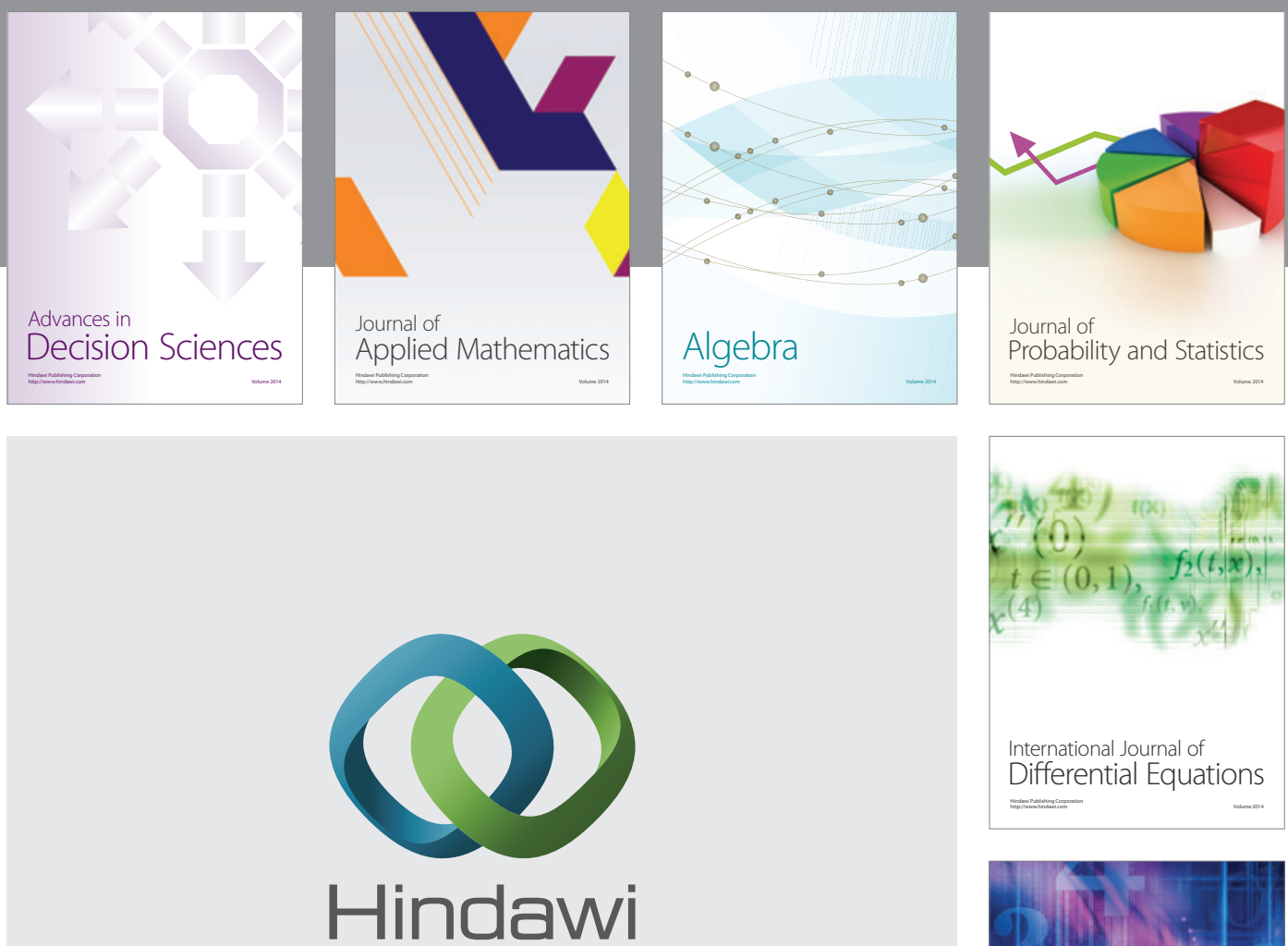

Submit your manuscripts at http://www.hindawi.com
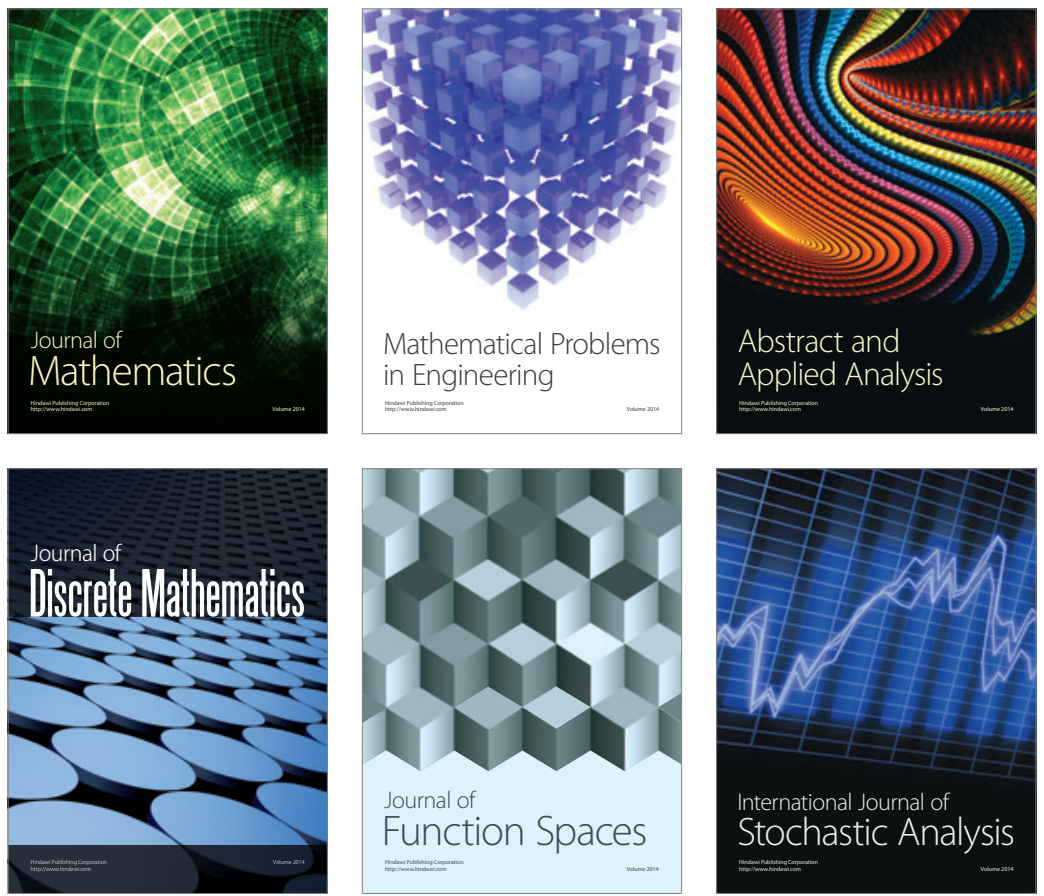

Journal of

Function Spaces

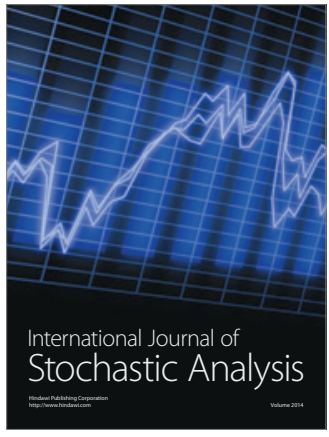

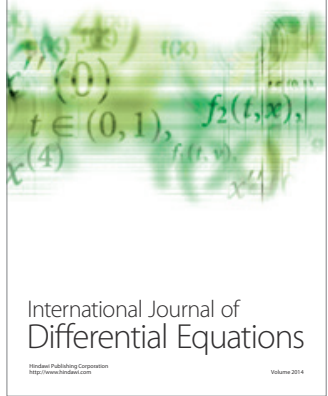
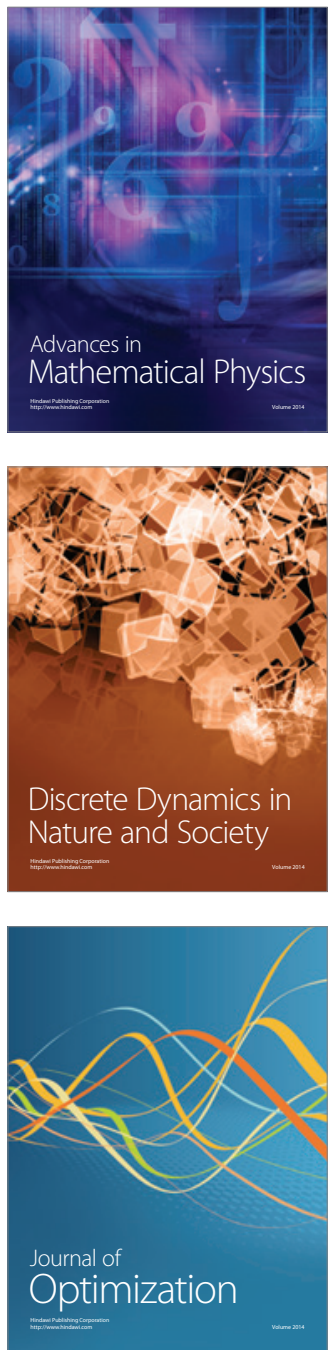